\section{ON INDICATIONS FOR IMMEDIATELY OPENING THE ABDOMEN IN ACUTE CASES."} BY

W. G. SPENCER, M.S , F.R.C.S. SURGEON TO THE WESTMINSTER HOSPITAL.

I propose to consider this subject especially from the point of view of country practice. It is one of those questions which is most prominent at the present time, for the all-sufficient reason that upon it turns in many cases the life or death of the patient.

If any one should ask why such cases come now so frequently into question, I have no doubt as to the answer-it is simply this, that in former days they were overlooked. In such cases a name was given as tho diagnosis, either a general term, or the case was labelled on account of some symptom to which attention was chiefly drawn. It was sought to relieve the patient's symptoms, and the disease was left to chance. If indeed such a case were submitted to a post-mortem examination, the record did not distinguish cause from effect, so that no emphasis was laid upon what might have been done at an early stage of the affection. Of this explanation, that the cases to be referred to were formerly overlooked, one might occupy the rest of the paper in recounting instances. I recall an excursus in Dr. Matthews Duncan's lectures on midwifery when he came to tell us that the fetal heart was first heard about 1820. He put his finger on the tip of his nose, and with more Scots brogue than usual declared to the theatre full of students that there were things in front of our noses which we could not then see.

A preliminary point which I propose to consider in a brief manner is the adaptability of modern surgery, as applied to abdominal cases, for practice in private houses and small hospitals.

The most advanced type of modern surgery is carried on in magnificently equipped institutions at a lavish expenditure both as regards foundation and maintenance. So marked is this the case that the London hospitals can no longer claim to represent the most advanced ideals. In former days they depended largely upon endowments, property held as capital after the death of donors, and only the interest expended. Of late years property received from the same class of donors has been treated as income. But the State has come to demand more and more from the same sources as have hitherto maintained the hospitals. And the London hospitals are apparently approaching a change when they must come under the State and the municipality; then the proverb will apply that he who pays the piper may claim to call the tune.

But if the larger ideals as to surgical installations come to be deemed essential, a serious bar to the proposition that abdominal surgery can be well carried out in private houses and hospitals with limited supplies will be formed. Of course there is ample reason for a large expenditure on modern requirements in a great hospital crowded with all sorts of cases. The most costly suggestions of modern bacteriology can be justified when one case follows close upon another into the operating theatre day after day.

But when one sets out to examine narrowly all details so as to pick out the essentials of modern surgery, we onght to read over again Lord Lister's instructions, for it will then be found that the methods of Listerian surgery adapt themselves to the exigencies of private and small hospital practice. And I may quote as the most recent anthority on this point the lectures recently published by M. Lucas. Championnière.

Whether a surgeon is studying how to furnish a small hospital on limited supplies, or whether he is trying to include in a bag all the things indispensable at an abdominal operation, the Listerian methods, with small additions, will form an efficient protection against germ infection. A surgical practice based on Lord Lister's latest instructions has anply proved itself to be safe and

* A paper read at the meeting of the West Somerset Branch of the * A paper read at the meeting of the West Somerserset Hospital on November 2nd, 1909 .

t Reviewed in the British Medical JournaL, 1909, vol. i, p. 793. successful anywhere and always. The ideals which are set forth in some new and magnificent hospitals, if they came to be regarded as universally essential, would put an end to all surgery except in the largest hospitals and costliest nuxsing homes.

Abdominal Conditions Requiring Immediatr Operation.

Passing on to the various conditions for which an immediate operation is required in abdominal cases, I will note a few points for each which, in my experience, have come to appear important.

Hernia.

First as to external hernia, and the possibility of a small irreducible femoral or umbilical hernia being missed, I have known men, keenly alive to the early recognition of surgical cases, yet who, in the rush of work, have taken the patient's complaint of occasional vomiting as a symptom to be treated without, at the moment, making an examination. The patient has omitted to complain of the hernia owing to its being an old-standing one and painless, so that when I came to operate there was advanced strangulation. More than one housesurgeon has reported the admission of an acute abdominal case without examining the hernial rings.

Practically it is always advisable to operate upon an irreducible femoral or umbilical hernia, and to do so immediately that it occasions vomiting, however slight. Pain and tenderness are further signs, but both may be absent until strangulation is far advanced.

The inguinal hernia which should be operated upon at once is that which comes down suddenly in a strong man and causes marked pain. There is the further reason for operating that the hernia may be cured. I would disconntenance any palliative measure, whether taxis or the hot bath. In a child, unless late of course, the application of an ice poultice almost invariably leads to the reduction of the hernia, and the question of operation may be postponed. In an old man with an inguinal hernia of long standing, which bas become irreducible, a hot bath should be given, with all watchfulness against faintness, after which a moderate application of taxis is indicated. But if taxis is not speedily successful an incision should be made without any further delay.

\section{Appendicitis.}

One is sorry to read that some physicians still maintain the confusion over the name "appendicitis" by using it in a double sense. There is the disease which the surgeon means by "appendicitis." We read that at Budapest physicians spoke of the medical treatment of appendicitis. If they were referring to what the surgeon calls appendicitis, then their treatment being of the harmless kind their cases were left to chance. If the cases were not what the surgeons call appendicitis, the proper general term in the absence of any further definition should be that of " colic."

A surgeon means by "appendicitis" a disease which begins by a retention somewhere in the lumen of the appendix, favoured by which the organisms in the retained material grow outwards through the wall of the appendix with all possible variations as to virulence and rapidity. When this inflammation reaches the peritoneal coat of the appendix the symptoms of appendicitis show them. selves for the first time, so that the symptoms of appendi. citis are those of infective peritonitis, it may be merely the peritoneum covering the appendix, or the peritoneum in the immediate neighbourhood, or wider still.

There is no medical treatment which can possibly have any influence upon the course of the disease. The communication with the caecum has been shut off from the beginning; the communication with the circulation is simply through a small artery: the only conceivable medical treatment would be an antitoxin, and that could hardly reach such a secluded spot, even if one had an antitoxin of the kind which would neutralize the toxins.

Appendicitis is the most frequent of surgical affections, and, like other surgeons, my experience runs into hundreds of cases. I am wholly in favour of operating as soon as the diagnosis is made, and of always removing the appendix. The mortality is then less than 5 per cent., some of which is made up by cases which present no signs until it is too late. 
The first sign of the disease, acute pain with vomiting, does not mark the commencement of appendicitis, but its result-peritonitis. This pain may be referred elsewhere than to the appendix region, when we must diagnose the case as one of acute infective peritonitis, for which an exploratory operation is desirable. That it is the appendix which is involved is for the moment only a presumption. This we shall the more readily make in the region of the appendix, if by percussion we can note an impaired resonance, either over the ileo caecal region or towards the ascending colon. This impairment of resonance indicates septic oedema, which spreads out in the subperitoneal tissues from the appendix, along with effusion into the peritoneal cavity. If the appendix is hanging over into the pelvis, then the impaired resonance is lower down, or one can only reach and feel the oedema and peritoneal effusion per rectum. In such an acute case the diagnosis of appendicitis is presumptive-the pain is too acute and general for us to feel the actual appendix. We must be keenly alive to the first signs of infective peritonitis. If we inspect the abdomen, and there is no sign of peristalsis when the abdomen is exposed, we may conclude that we have not to deal with colic. If we observe peristalsis, we have not to do with peritonitis, but with colic or some form of intestinal obstruction.

We should in abdominal cases pay no primary attention to the patient's temperature. The pulse will certainly be affected, but we must not wait if other signs are present. The pulse will slowly rise to a rate above 100 , and have the peritoneal characteristics. But it does not make this rise in some cases until late. Even in an acute attack the pulse may be slower than 80 for a time, probably owing to primary shock.

Where the pain is not very acute and general, we can usually get localizing signs; sometimes it is the patient himself who refers it to McBurney's spot, or the neighbour hood. More surely the surgeon learns to feel the inflamed appendix through the abdominal wall, and the patient confirms the surgeon's sense of touch by complaining of a particular tenderness.

Although I have followed consistently the plan of operating at once, I cannot recall that I have made an error by operating in acute cases. If $I$ have not found appendicitis, I have exposed some other condition, of which I could mention a long list, all in some way amenable to surgical treatment.

Really the operations on the appendix which may perhaps be exposed to criticism are those done when the patient has had recurring attacks of some sort, but without a thickened or painful appendix being distinguishable. Then one may find the appendix kinked, and perhaps an infection of the wall of the appendix can only be demonstrated on microscopical examination. But there remain a few cases in which the operation has been done on account of obscure symptoms, in which the appendix neither appeared kinked, nor could any marked change be found on microscopical examination. I suppose such exceptions may have been instances of appendicular colic in the true sense of the term, not appendicitis, but a form of enterospasm.

Returning to acute cases, I would especially emphasize the necessity of being beforehand in the case of boys and girls, or the most heart-breaking calamity may occur. One very painful spot, with a little impaired resonance around, in a schoolboy who has vomited once and looks ill, are about all the signs you may meet with of acute gangrene of the appendix. Eren suppose one should operate unnecessarily in a case, it is by a set determination to operate early that many lives are saved; to any caviller one should repeat the American saying, "The real lifesaving operation is the operation within the first twenty. four hours of the first attack."

Gastro.duodenal Ulceration and Perforation.

When one passes to the second group of acute abdominal cases-those in connexion with gastric and duodenal ulceration-a surgeon cannot express such dogmatic opinions as about appendicitis, because he has not suff. cient experience of early cases, whether of dyspepsia or of ulceration. I have seen with physicians a number of patients with acute haematemesis, and in all we have agreed that the patient should be treated medically; of these cases all have recovered from that attack except one; of those that have recovered some have then declined gastro-jejunostomy, others have had gastro-jejunostomy performed, when I have been confirmed in the view that I could have done nothing by immediate interference. The patient who died was seen by $m \theta$ in consultation several times during the month she lived, but there was never the slightest chance for an operation. The haemorrhage stopped, but she gradually sank with what must have been a deep ulceration. Two cases died after gastrojejunostomy, the haemorrhage had kept on recurring in small amount, and at last the physician in each case recommended operation as a last resource. The operation was done quickly, and neither patient seemed any worse for it, but the haemorrhage returned in about three or four days, and death followed.

As regards the cases of acute perforation, if there has been a distinct history of gastric trouble, a sudden attack of acute pain in the epigastrium with more or less collapse, and if one can assure oneself that there is commencing peritonitis, there is no occasion to wait. In a case in which these special points are rague, the one important thing is that the patient should have nothing by the mouth. It is a grievous drawback to open the epigastrium, and for food and brandy to pour out. If the patient is put to bed, given rectal saline infusion and nutrient enemata, but no morphine, one can then watch for the appearance of definite indications. The steady rise of the pulse, the localization of pain in the epigastrium, the commencement of distension of the epigastrium by gasthese signs will appear in a few hours. Owing doubtless to the antiseptic properties of the hydrochloric acid in the gastric juice the infective peritonitis is not so virulent as in appendicitis, so that a perforation, especially behind, may slowly develop a subdiaphragmatic abscess and give more time for making a diagnosis. The most urgent. operation, such as that for perforation of a duodenal ulcer, is usually plainly indicated by the acute localized peritoneal pain due to the duodenal contents coming in contact. with healthy peritoneum.

\section{Gangrene of the Gall Bladder.}

An immediate operation is required in the case of acute gangrene of the gall bladder, or the patient will die. In the cases I have seen there has been a distinct history of biliary trouble, and an enlarged gall bladder may have been felt beforehand. The attack is that of acute infective peritonitis below the ninth rib on the right side causing. pain, rigidity, impaired resonance and soon brawny induration of the abdominal wall. The temperature is likely to begin with a rise, even a rigor, but, as in other cases of gangrenous inflammation, the later tendency of the temperature is to fall. Moreover, if there is jaundice, and according to the extent of the jaundice, the pulse may be kept from increasing in rate until later than would accord with the amount of infective inflammation.

In the cases I have seen the local indications $i$ for. operation were evident. If there is delay, there $\mathrm{s}^{\prime} \mathrm{a}$. double chance against the patient, not only from the infective peritonitis; but also from the extension of the infective inflammation to the branches of the portal and hepatic vein and the inferior vena cava, and along the hepatic ducts into the liver. I have removed the gall bladder and gall stones and plugged the wound with gauze, and the cases have done well.

\section{Acute Pancreatic Abscess.}

A dangerous but rarer condition is that of acute pan: creatic haemorrhage and abscess, which may resemble either a duodenal perforation or gangrene of the gall bladder. If found, the only thing to do is to plug with gauze and end the operation as quickly as one can, for the cases are most fatal.

\section{Pelvic Cases in Women.}

Of the acute pelvic cases in women in which an imme diate operation is necessary, perhaps the easiest to diagnose is a twisted ovarian cyst, because of the acute symptoms and the existence of the cystic swelling. Also there is no difficulty in deciding to operate for suppuration supervening upon an ovarian dermoid, salpingitis, or haematocele. As regards extrauterine fetation, fortunately the necessity for an immediate operation is exceptional The case would now generally be recognizable after the 
early rupture into the layers of the broad ligament, and be operated upon in the interval, before there is danger of further rupture. When rupture into the peritoneal cavity does occur, the signs which have been described by Lawson Tait and Cullingworth appear-acute abdominal tendercess and rigidity, increasing anaemia, and the collection of free fluid in the peritoneal cavity. If the surgeon operates early in the case of a primary rupture into the peritoneum, he quickly clamps the bleeding point and goes on to infuse saline fluid, and the case may be expected to recover rapidly. When there is a late rupture into the peritoneal cavity, the surgeon is in terrible difficulties. I had to operate a while ago on such a case; she had come a good distance to the hospital; the history was clear enough, also the signs, and she was acutely anaemic. I found blood-clot widespread, and mixed up with intestines, to which the placenta adhered. I could only clear out the fetus, and part of the clot and membranes, then plug the sac with gauze. She soon died.

I may mention two rarer sorts of cases which I have had to operate upon, because in neither, for more than one reason, may any history be forthcoming.

The first group are due to streptococcal infection, in connexion, one has to presume, with attempts at artificial abortion. There is, of course, a history in cases of regular labour and miscarriage. What is evident is an acute infective pelvic peritonitis, great pain and tenderness, with impaired resonance over the hypogastrium. By the vagina and rectum one can make out some uterine enlargement; the incision through the lower part of the linea semilunaris on either side will open up a parametric abscess, if there is one, without entering the peritoneal cavity. This I have had to do several times; or the peritoneum is opened up, and there is exposed a collection of turbid fluid around the enlarged suppurating or semi gangrenous uterus. In one case I passed a tube from the hypogastrium through the rotten uterus out by the vagina. I think the patient might have lived, so far as the local condition was concerned, but she developed pericarditis and died. The case was in a private house, and the practitioner only knew part of the history. In a similar case, which died in hospital, the marks of infected punctures were found on the cervix uteri. In a third case, which lived, gauze was packed round the infected uterus.

The other set of cases in which one may get no history is that of acute gonorrhoeal pelvic peritonitis in girls and married women. I have operated on the supposition that the cases were appendicular, and have found the tubes inflamed and pus escaping, the appendix being free, or, it may be, also involved. One seeks to confirm the diagnosis by examining for gonococci. Beforehand, by rectal examination one finds evidences of pelvic peritonitis, but there is no circumscribed swelling as in the cases of tubal and ovarian suppuration.

The cases above referred to all have this in common, that the sign for immediate operation is marked by the onset of acute infective peritonitis at some one point in the peritoneal cavity.

\section{Intestinal O'sstruction.}

The other great group of cases have as the common sign intestinal obstruction.

The most difficult of all the abdominal cases with which the surgeon has to deal are those in which the symptoms of intestinal obstruction are followed by the signs of general infective peritonitis. But such a case need not be discussed with regard to the question of immediate operation. That goes without saying; it is the prognosis which is so bad. Is the case then hopeless, and operation contraindicated? No, unless the patient is beginning to die. Rapid operating, emptying the intestines, sponging out the peritoneum, accompanied and followed by continuous saline infusion, have led of late to wonderful recoveries from a formerly hopeless condition. The chief aim in all cases of intestinal obstruction is to operate before the infective peritonitis has supervened upon the obstruction.

Intussusception is common among weak, ill-fed children. Fortunately surgeons now see the children early when the tumour can usually be detected, although as to this there may be some obscurity with the tumour in the hepatic region. But with the evidence of the acute onset and a feeling of the tumour, one should not think of waiting for further signs, such as blood and mucus in the rectum. The one chance of success is to operate at once; as the abdomen gets distended the tumour is less easily felt, and the increased peristalsis of the bowel is less evident, but we now find blood and mucus in the rectum. Students are apt to catch at some minor point because it is new, and they mention the lack of fullness in the caecal region, after all a negative point, and very easily obliterated by the tympanites. The most dangerous type of intussusception is that which occurs in the course of Henoch's purpura; the case being under treatment for purpura, intussusception may not be noticed. In one upon which I operated for my colleague, Dr. Hall, although early and readily reduced, the case died. Fortunately most cases of intussusception, if they can be operated upon within a few hours of the onset, will get well.

In most of the cases of intestinal obstruction we can make a guess at the cause, and should operate before the acute stage of distension. It is most important to explore, for by so doing we can remove the disease and cure the patient. Even although we find some irremovable cancer, there are opportunities of anastomosis or of appendicostomy which may save the patient from an artificial anus. Thus in a recent case there was an abscess around an irremovable sigmoid cancer which had caused an attack of partial obstruction; an appendicostomy has sufficed to prevent another attack of obstruction.

When the bowels are already distended the one essential to success is to empty them by siphoning off the contents. But if there is delay until, in addition to the distension, there is already peritonitis, the muscular wall of the bowe may not recover even although the gut has been emptied.

The case of intestinal obstruction which gets put off too long is the patient who only vomits occasionally and perhaps has only foul borborygmi. His abdomen shows coils of intestines partially distended with marked peristalsis; such a case if left too long will be seized with collapse and sudden tympanites, and then operation will be too late.

Abdominal Injuries.

I have had no experience of importance with accidents. No English surgeon has had any number of cases of ab. dominal wounds from pistol bullets. We must rely upon the experience of American surgeons. Every case of pistol bullet wound must be explored at once, the track followed up, the damage repaired, and then a drain must be inserted.

In cases of abdominal contusions the first thing to do is to treat the patient for the shock, give nothing by the mouth, no morphine, and to examine and watch most closely for special indications. Mr. Rock Carling and also Mr. J. Berry have had successful cases of rupture of the jejunum close to the duodenum, the cardinal sign being the acute and localized pain, which, starting at the time of the accident, increases in severity. This points to an immediate operation.

Then there are the special signs of rupture of the various organs which may present themselves as the case is closely watched.

Caesarean Section.

When I think of other reasons for immediately operating in abdominal cases such as may be met with I feel disposed to praise Caesarean section, for within my limited experience it seems to me a straightforward abdominal experience it seich one can be confident of success if it be done like other abdominal operations, early, before the mother is in any way infected or the child injured. One thinks that perhaps the babies in lying-in hospitals may rometimes seem to students rather superfluities. But in private practice the outlook is very different, for many of the women may have but one chance in their lives of being a mother. That is the aspect which weighs so heavily for early Caesarean section and so unfavourably against the alternative courses.

Caesarean section will come more and more into practice by anyone who can do other abdominal operations, and not only for pelvic obstruction. Dr. Griffiths has adopted it successfully for placenta praevia, and although I have never seen a case of placenta praevia, the difficulties and dangers of acute haemorrhage are well known. I recall that when your president's brother was my house-surgeon an experienced practitioner noted 
at once that a woman, to whom he was called at the first commencement of labour, had a small male type of pelvis, and be sent her up to me at the hospital forthwith. I operated at once; she made a recovery, as aseptic abdowinal cases do. She has frequently been up to the ward with her child; she has not been again pregnant, and so may be one of the women alluded to above, who have but one opportunity.

Renal Obstruction and Suppression of Urine.

If we may include renal among abdominal cases, then suppression, or threatened suppression, of urine will form the chief indication for immediate operation when we can find evidence that the cause is obstructive. An $x$-ray examination may show calculi on both sides, or even slight distension of the kidney and pelvis. In the absence of an $x$-ray examination, one must rely upon the history of the case and upen palpation. But, if there is any suspicion, in face of suppression, that the cause is an obstructive one which will not yield to medical treatment, early nephrotomy should be done. If done early, one may do it on one side first, and wait before cutting into the opposite kidney.

\section{ON THE USES OF CATGUT IN SURG ERY: \\ WITH SPECIAL REFERENCE TO IODINE CATGUT AND IODINE-FORMALIN CATGUT.}

By C. YELVERTON PEARSON, M.D., F.R.C.S., PROPESSOR OF SURGERY, ONITERSITY COLLEGE, CORK.

Considrring the advances that have been made of recent years in the preparation of catgut by improved and simplified methods, it is strange that such diversity of opinion should still exist amongst surgeons of repute regarding its safety and general applicability for surgical purposes. At the present time operating surgeons adopt one of the following attitudes regarding its use

1. There may be some who never employ it.

2. There are those who employ it to a very limited extent, and chiefly in infected wounds.

3. There are others who, eitber owing to some unfarourable experience or to the reports of tetanus and other disasters in the practice of other surgeons, use it with a feeling of uncertainty and anxiety.

4. Finally, there are those who use it freely in all cases where such a material is suitable.

As a striking example of such diversity in practice I may mention that during a recent visit to the United States I found that in the Mayos' Clinic at Rochester, Minn., iodine catgut was extensively employed for ligatures and buried sutures, to the complete exclusion of other materials, save where some more resisting substance is necessary-for example, chromicized gut is used for hernia operations and for the inner row of gastro-intestinal sutares, the external row consisting of iron.dyed Irish linen. The iodine catgut used is sterilized by Willard Bartlett's method, ${ }^{1}$ which consists in heating it in liquid petrolatum previous to its being placed in a 1 per cent. solation of iodine crystals in Columbian spirits. This preliminary sterilization is somewhat troublesome and quite unnecessary for the preparation of iodine catgut, as I shall presently point out. The use of catgut so prepared has, however, given the greatest satisfaction; it has been used by the brothers Mayo and their assistants in over 5,000 operations with most satisfactory results, and has been adopted by Murphy, Deaver, and others.

In the surgical clinic of Johns Hopkins Hospital, on the other hand, I found that catgat is used to only a very limited extent, iron.dyed silk, freshly boiled, being extensively employed for ligatures and buried satures. Dr. Finny informed me that he avoided the use of catgut as far as possible, owing to the tronble to which it frequently gave rise, notwithstanding its most carefal preparation by various methods; the process which still found most favour there being the difficult and cumbersome cumol method.

It is my belief that so long as surgeons continue to employ a simple aseptic catgut, no matter what the pro cess of sterilization employed, so long must they expect to have trouble and anxiety with their operation wounds.
I have elsewhere ${ }^{2}$ taken considerable pains to point out the objections to the use of plaini sterile catgut, and insisted on having it impregnated with some reliable antiseptic. This is one of those exceptional instances in which antisepsis as opposed to simple asepsis possesses a distinct advantage.

Ewald found that catgut has the power of exciting sappuration, even when it is perfectly sterile; this property he attributed to an unknown toxic substance. The experiments of Klemm led him to believe that though catgnt may be sterile, it affords a suitable soil for the cultivation of micro-organisms. Apart, however, from infection, as I have pointed out, "it appears that catgut sometimes possesses chemotactic properties which lead to the formation in the tissues of sterile pus," 8 or, at least, a fluid which differs only from true pus in the absence of pyogenic micro-organisms.

During the past few years several cases of postoperative tetanus have been reported, and in some at least of the cases catgut has been held responsible, and, I believe, not unjustly, though I do not know what kind of catgut was used. What I desire specially to point out is that, even assuming the catgut to be sterile but devoid of antiseptic properties, $I$ think it not only possible, but highly probable, that if placed in a favourable situation in the peritoneal cavity, owing to its chemical properties it might attract to it micro organisms from the intestinal canal such as $B$. coli, $B$. enteritidis, or possibly some organism which, though not identical with the $B$. tetani, may be capable of developing a tetanic toxin.

Now, if catgut be not alone sterile, but also impregnated with some germicidal substance which protects it from infection, this could not occur. It is not enough that the gut is incorporated with one or another antiseptic; the antiseptic itself must possess certain qualities to be ideal. It must be strongly antiseptic, preferably germicidal, so that it not only protects the gut from infection bat also sterilizes its track; it must be able to penetrate the gat throughout, without injuring its physical properties; be non-toxic and non-irritant; not interfere with timely absorption and must make the catgat antiseptic throughout its entire active life

So far, the substance which has given for this purpose most general satisfaction is iodine, which, though powerfully germicidal as well as antiseptic, does not, when brought into contact with the deeper tissues in solutions of moderate strength, seem to exert any injurious influencet Nor have I ever seen or heard of a case of iodism from the free use of catgut impregnated with it, which shows that it is not rapidly absorbed.

\section{Iodized Catgat.}

The method of preparing iodized catgut which first gained it popnlarity was that of Claudius, which con. sisted in immersing the gut in a 1 per cent. watery solution of iodine and potassium iodide for at least eight days. It was said that the catgut might be kept in this solution for a considerable time without deteriorating in quality. This I soon found to be a fallaey, and at the same time satisfied myself that loss of tensile strength was not due to the evaporation of the iodine, as suggested by Mr. A. S. Barling, ${ }^{4}$ but to the chemical action of the water on the fibrous tissue. This softening by simple maceration will be found to take place in fibrous tissue when it is immersed for any considerable time in distilled water, in the absence of all putrefactive changes and even in the presence of some antiseptics, but will not occur in the presence of various hardening agents-and more especially alcohol, which has the further advantage that it does not produce brittleness.

It was owing to the recognition of this action of watery solutions of iodine on catgat that I was led to use different methods for its preservation, three of which are described in my work on Modern Surgical Technique as being satisfactory-namely:

(1) The addition to the aqueous iodine solution of an equad quantity of alcohol; (2) the addition to it of an equal quantity of 5 per cent. phenol solution, which, by combining with the iodine, removes most of the colour, but gives an antiseptic catgut that will keep admirably; (3) transferring the catgut to catgut that will keep admirably; (3) transferring the catgut to a spirituous preserving solution of mercuric biniodide, having
previously washed it with phenol solution to remove excess of previously washed it with
lodine from its surface. 